\title{
Decay dynamics of quantum dots influenced by the local density of optical states of two-dimensional photonic crystal membranes
}

Julsgaard, Brian; Johansen, Jeppe; Stobbe, Søren; Stolberg-Rohr, T.; Sünner, T.; Kamp, M.; Forchel, A.; Lodahl, Peter

Published in:

Applied Physics Letters

Link to article, DOI:

$10.1063 / 1.2977605$

Publication date:

2008

Document Version

Publisher's PDF, also known as Version of record

Link back to DTU Orbit

Citation (APA):

Julsgaard, B., Johansen, J., Stobbe, S., Stolberg-Rohr, T., Sünner, T., Kamp, M., Forchel, A., \& Lodahl, P. (2008). Decay dynamics of quantum dots influenced by the local density of optical states of two-dimensional photonic crystal membranes. Applied Physics Letters, 93(9), 094102. https://doi.org/10.1063/1.2977605

\section{General rights}

Copyright and moral rights for the publications made accessible in the public portal are retained by the authors and/or other copyright owners and it is a condition of accessing publications that users recognise and abide by the legal requirements associated with these rights.

- Users may download and print one copy of any publication from the public portal for the purpose of private study or research.

- You may not further distribute the material or use it for any profit-making activity or commercial gain

- You may freely distribute the URL identifying the publication in the public portal 


\title{
Decay dynamics of quantum dots influenced by the local density of optical states of two-dimensional photonic crystal membranes
}

\author{
B. Julsgaard, ${ }^{1, a)}$ J. Johansen, ${ }^{1}$ S. Stobbe, ${ }^{1}$ T. Stolberg-Rohr, ${ }^{1}$ T. Sünner, ${ }^{2}$ M. Kamp, ${ }^{2}$ \\ A. Forchel, ${ }^{2}$ and P. Lodahl ${ }^{1, b}$ \\ ${ }^{1}$ DTU Fotonik, Department of Photonics Engineering, Technical University of Denmark, Ørsteds Plads 343, \\ 2800 Kgs. Lyngby, Denmark \\ ${ }^{2}$ Technische Physik, Universität Würzburg, Am Hubland, D-97074 Würzburg, Germany
}

(Received 25 February 2008; accepted 13 August 2008; published online 2 September 2008)

\begin{abstract}
We have performed time-resolved spectroscopy on InAs quantum dot ensembles in photonic crystal membranes. The influence of the photonic crystal is investigated by varying the lattice constant systematically. We observe a strong slow down of the quantum dots' spontaneous emission rates as the two-dimensional bandgap is tuned through their emission frequencies. The measured band edges are in full agreement with theoretical predictions. We characterize the multiexponential decay curves by their mean decay time and find enhancement of the spontaneous emission at the bandgap edges and strong inhibition inside the bandgap in good agreement with local density of states calculations. () 2008 American Institute of Physics. [DOI: 10.1063/1.2977605]
\end{abstract}

Photonic crystals (PCs) are of fundamental importance due to their ability to modify the dynamics of light-matter interaction. ${ }^{1}$ The radiative lifetime of internal light sources can be controlled and the spatial emission pattern can be modified. ${ }^{2-5}$ These effects open up the possibility of improving the efficiency and compactness of devices such as lightemitting diodes (LEDs), ${ }^{6}$ and offer control of the propagation of single photons of use in, e.g., quantum information protocols. ${ }^{7}$ In order to determine the ultimate potential of PCs in such applications, a thorough understanding of light emitters in PCs is needed. Here we study systematically the spontaneous emission dynamics of quantum dots (QDs) in two-dimensional (2D) PC membranes (PCMs) and compare our results to theory. The radiative decay rate of a single QD inside the PCM is governed by the projected local density of optical states (LDOS) ${ }^{8}$ In order to probe variations herein, we investigate ensembles of QDs since in this case any dependence on the property of individual QDs is averaged out. Furthermore, QD ensembles provide good signal-to-noise ratio in the measurements and are important for large-volume applications such as PC lasers, LEDs, or solar cells. The projected LDOS varies with the position and orientation of the emitter inside the $\mathrm{PCM},{ }^{9}$ and a distribution of decay rates is expected to arise from the QD ensemble with a multiexponential decay curve as a result. Such an effect has already been reported in three-dimensional (3D) inverse opals using colloidal QDs, ${ }^{10}$ but so far a systematic study of the decay curves in 2D PCMs exists only theoretically. ${ }^{9}$

We consider ensembles of InAs QDs in GaAs PCMs that have been fabricated with systematically varied lattice constants. We optically excite the QDs within the membranes and detect the time-resolved spontaneous emission from a spectrally selected ensemble of approximately $6 \times 10^{3}$ QDs. The dimension of each membrane is $40 \times 40 \mu \mathrm{m}^{2}$ with thickness of $150 \mathrm{~nm}$. The QDs are grown by molecular beam epitaxy with density of $\approx 300 \mu \mathrm{m}^{-2}$ and centered within the membrane. The PCM consists of holes arranged in a trian-

\footnotetext{
${ }^{a)}$ Electronic mail: brianj@phys.au.dk.

${ }^{b)}$ Electronic mail: pelo@fotonik.dtu.dk.
}

gular lattice, see Fig. 1(a), with hole radius $r$ and lattice constant (hole spacing) $a$. All membranes in this experiment are in the range $r / a=0.313 \pm 0.006$ measured by scanning electron microscopy (SEM), and the lattice constant ranges from 180 to $470 \mathrm{~nm}$ in steps of $10 \mathrm{~nm}$. The samples are placed in a closed-cycle cryostat keeping the temperature at $14 \mathrm{~K}$. The QDs in the PCMs are excited in the GaAs barrier with a PicoQuant PDL-800 pulsed diode laser running at 781 $\mathrm{nm}$ with a repetition period of $100 \mathrm{~ns}$. The chosen pump intensity corresponds to an upper estimate of five to ten electron-hole pairs per QD generated in each excitation pulse, and emissions from the ground state, the biexcitonic state, and the higher excited states of the QDs are thus expected. Spectral selection of the ground state energy $(\lambda$ $=980 \mathrm{~nm}$ ) ensures that we only observe spontaneous emissions from the ground and biexcitonic states. Saturation of the QDs leads to a $50 \%$ increase in the decay rate, but this effect is expected to be independent of the PCM geometry and therefore does not pose a problem to our measurements. The high pump power improves the signal-to-noise ratio

(a)

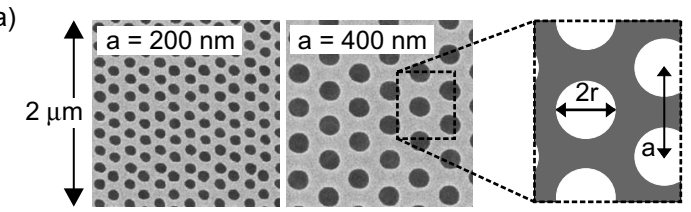

(b)

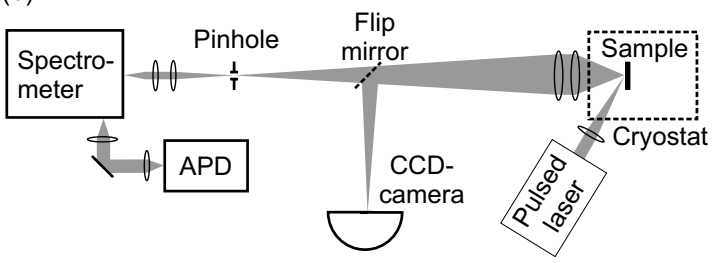

FIG. 1. (a) SEM images of the PCMs. (b) Experimental setup: The PCMs are placed in a cryostat and excited by a pulsed laser source. The timeresolved spontaneous emission is detected by an avalanche photodiode after spectral selection. A pinhole selects the emission from the central part of the PCM, the position of which can be adjusted with assistance from a charge coupled device camera. 

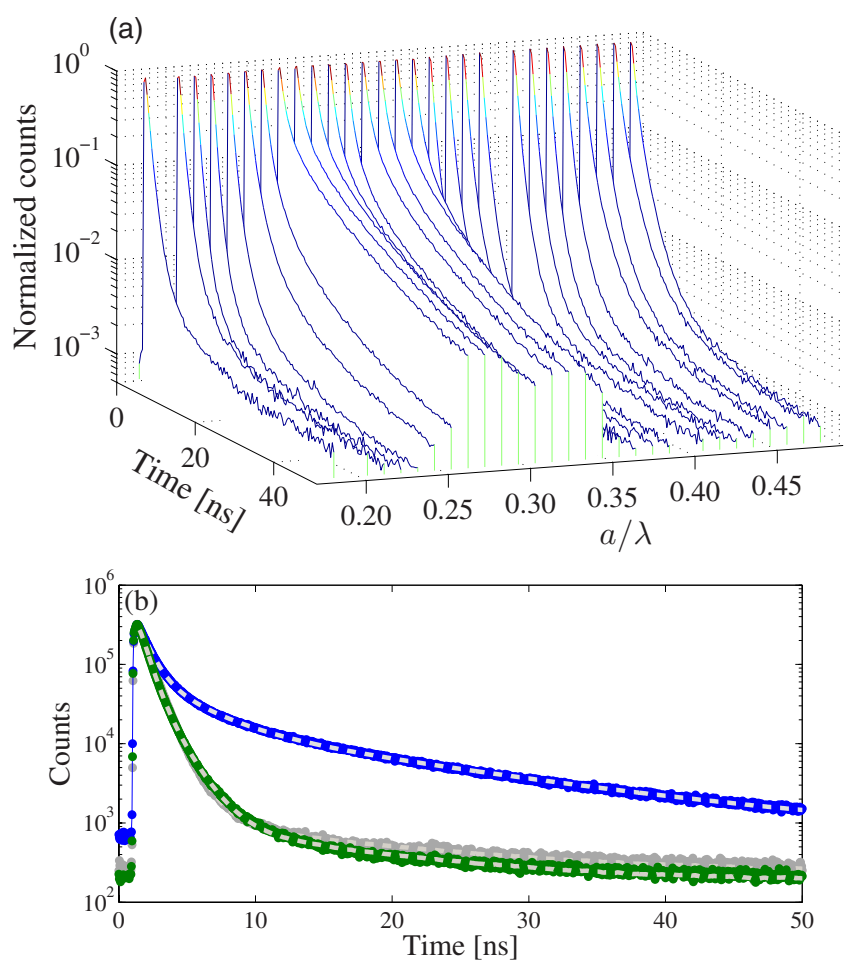

FIG. 2. (Color online) (a) Time-resolved decay curves shown on a normalized scale for varying lattice constant. For clarity, only the first $50 \mathrm{~ns}$ are displayed. A $2 \mathrm{D}$ bandgap effect is clearly visible in the region $0.26 \leq a / \lambda$ $\leq 0.35$. (b) Fit of the triple-exponential model $f(t)$ (dashed lines) to the measured decay curves for $a / \lambda=0.296$ (upper blue curve) and 0.388 (lower green curve). For comparison, the decay curve of an unpatterned sample is also shown (gray curve slightly above the lower curve). All curves are scaled to have coincident maxima.

compared to the case of nonsaturated QDs. The experimental setup is shown in Fig. 1(b).

The recorded decay curves are shown on a normalized scale for comparison in Fig. 2(a) with varying lattice constant $a$ and fixed detection wavelength $\lambda=980 \mathrm{~nm}$ within a bandwidth of $2 \mathrm{~nm}$. We readily observe a pronounced slow down of the decay dynamics in the range of $0.26 \leq a / \lambda$ $\leq 0.35$. This slow down is the signature of strongly inhibited spontaneous emission for emission energies within the 2D photonic bandgap. Note that the decay curves span up to three decades due to the long repetition period of the laser. This allows for a detailed study of the slow components of the decay curves where strong inhibition of spontaneous emission will appear.

As expected from the strong position and orientation dependence of the LDOS, ${ }^{9}$ the spontaneous emission decay curves are multiexponential. Approximating the initial fast decay by a single-exponential decay model will only determine the fastest decay rates in the multiexponential decay. Hence, a single-exponential model will clearly not suffice in characterizing the decay dynamics. A suitable measure to quantify the overall change in emission rate is the inverse of the mean decay time $\tau_{m}^{-1}$, which is computed as $\tau_{m}^{-1}$ $=\int_{0}^{\infty} f(t) d t / \int_{0}^{\infty} t f(t) d t$, where $f(t)$ describes the ensemble luminescence. To obtain $f(t)$ we fit a triple-exponential model to the data. The model has been convolved with the instrument response function ${ }^{11}$ obtained by detecting the pump laser scattered from the sample. Examples of triple-exponential models fitted to the data are shown in Fig. 2(b). We stress that the individual parameters are of no physical significance,
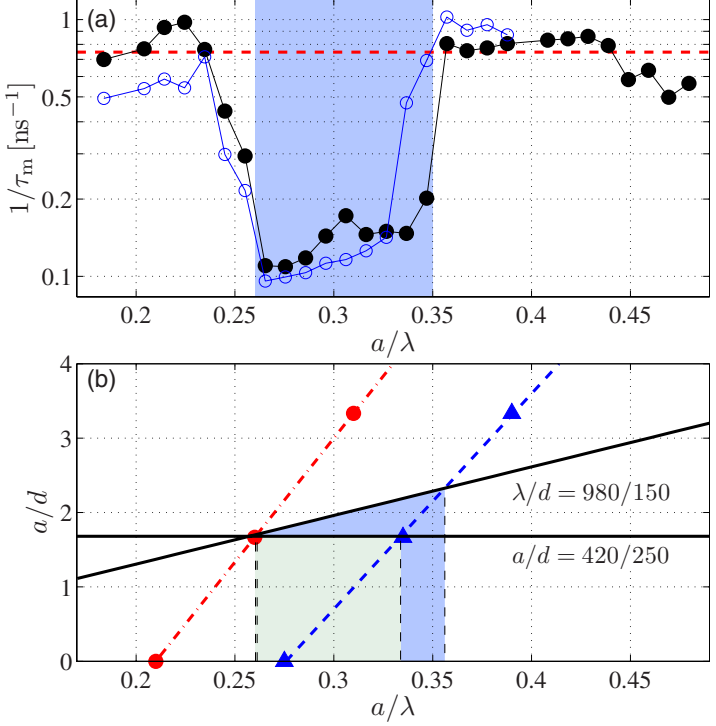

FIG. 3. (Color online) (a) The inverse mean decay time $\tau_{m}^{-1}$ of the experimental data (solid black dots) and the simulations (open blue circles). The dashed red line marks the value of $\tau_{m}^{-1}$ measured in absence of the PCM. The shaded region indicates the measured 2D bandgap. (b) The positions of band edges for various values of $a / \lambda$ and $a / d$. Red circles and blue triangles are taken from Ref. 15 with $r / a=0.3$ being consistent with the calculations of Koenderink et al., ${ }^{9}$ which are constrained to the horizontal line. The calculated $2 \mathrm{D}$ bandgap is indicated by the bright shading. Our experiment follows the inclined line with a broader 2D photonic bandgap as a result (dark shading).

the triple-exponential model is merely chosen as it is able to model the luminescence decay well and thus ensures a reliable extraction of $\tau_{m}^{-1}$.

The inverse mean decay times are plotted in Fig. 3(a) for the various values of $a / \lambda$. Compared to the value of $0.75 \mathrm{~ns}^{-1}$ obtained in absence of the PCM, we observe more than sixfold decrease in the inverse mean decay time inside the $2 \mathrm{D}$ photonic bandgap and an increase of up to $30 \%$ outside the bandgap. It should be noted that the effect of variations in the LDOS will be less pronounced in the mean decay time than in the purely radiative decay time since the former also includes contributions from nonradiative decay, which is unaffected by the LDOS. ${ }^{12}$ The experimental variations in the mean decay times are thus expected to be a conservative estimate of the actual LDOS variations in the PCM.

In order to compare the measured mean decay times with theory, the complex QD decay dynamics in a PC must be adequately accounted for. The decay curves for an ensemble of QDs will depend on the position and the orientation of the emitter through the LDOS, redistribution of the emitted light, and as well as internal QD dynamics. Simulation of the data is a comprehensive task and the details will be described elsewhere. Here we briefly discuss the approach and show the resulting comparison [Fig. 3(a)]. The radiative decay rate is modeled using the full 3D LDOS that was calculated for seven different positions and two orthogonal dipole orientations in Ref. 9. Redistribution is accounted for by assuming that modes propagating in the slab are completely inhibited by the 2D photonic bandgap, while radiation modes are unaltered. This simplistic model has been implemented successfully in the literature, ${ }^{3,4}$ however, a rigorous treatment would require calculating the full 3D Green's function of the PCM, which is not available in the literature. Finally, internal QD dynamics is modeled by including the interplay between 
bright and dark exciton states, which leads to a biexponential decay of the spontaneous emission. ${ }^{13,14}$ The relevant parameters describing the QD dynamics, including nonradiative decay $^{12}$ (which is assumed identical for all QDs and hence neglecting possible surface recombination effects at the membrane holes), were derived from measurements performed on an unpatterned membrane.

As shown in Fig. 3(a), we find a remarkably good agreement between experimental observations and simulated inverse mean decay times. This is to our knowledge the first detailed comparison between experiment and theory for QDs in PCs. Note in Fig. 3(a) that the measured spectral position of the upper bandgap edge deviates from the calculated value based on Ref. 9. However, this is readily explained by noting that the scaled frequencies of the band edges $a / \lambda$ vary linearly with $a / d$ as calculated in Ref. 15, where the spectral positions of the 2D photonic bandgap edges were determined for three different ratios between the lattice spacing $a$ and the membrane thickness $d$. This allows for interpolation into any value of this ratio, as shown in Fig. 3(b). The LDOS calculations in Ref. 9 were performed for a constant membrane thickness and lattice spacing $a / d=420 \mathrm{~nm} / 250 \mathrm{~nm}$, while varying the scaled emission frequency $a / \lambda$ corresponding to the horizontal line in Fig. 3(b). It can be shown that identical results will be obtained by scaling both $a$ and $d$ while keeping $\lambda$ fixed. Since it is very inconvenient to vary the membrane thickness $d$, our experiment is performed for fixed detection wavelength $\lambda$ and membrane thickness $d$, while the lattice constant $a$ is varied. In Fig. 3(b) this corresponds to the inclined line $a / d=a / \lambda \cdot \lambda / d=a / \lambda .980 \mathrm{~nm} / 150 \mathrm{~nm}$ which intersects the red (dash dotted) and blue (dashed) band-edge lines very closely (within experimental precision) to our observed values of 0.26 and 0.35 , respectively. While very good agreement between experiment and theory is obtained, when comparing the inverse mean decay time $\tau_{m}^{-1}$, as is apparent from Fig. 3(a), we note that increased complexity is found when comparing the full decay curves to theory (not shown). We believe that the discrepancies found are primarily caused by the rather simplistic model for the redistribution of light while deviations due to the slight differences in $d$ between experiment and theory are expected to be of minor importance.
In conclusion, we have carried out a systematic study of the spontaneous emission dynamics in 2D PCMs. Very pronounced inhibition of spontaneous emission was demonstrated within the range of lattice constants for which a $2 \mathrm{D}$ photonic bandgap is predicted by theory. Comparing to a reference measurement on QDs in unpatterned membranes, we report an inverse mean decay time that is reduced by more than a factor of 6 for emission energies inside the bandgap while an increase of $30 \%$ was observed on the red side of the bandgap. Our experiment was compared to theory taking into account the full 3D LDOS and very good agreement was observed.

We thank Femius Koenderink for generously sharing his LDOS calculations and Jørn M. Hvam for fruitful discussion and general support. We gratefully acknowledge financial support from the Danish Research Council (FNU Grant Nos. 272-05-0083 and 272-06-0138, and FTP Grant No. 274-070459). The work is part of the EU project "QPhoton." B. Julsgaard is supported by the Carlsberg Foundation.

${ }^{1}$ E. Yablonovitch, Phys. Rev. Lett. 58, 2059 (1987).

${ }^{2}$ P. Lodahl, A. F. van Driel, I. S. Nikolaev, A. Irman, K. Overgaag, D. Vanmaekelbergh, and W. L. Vos, Nature (London) 430, 654 (2004).

${ }^{3}$ M. Fujita, S. Takahash, Y. Tanaka, T. Asano, and S. Noda, Science 308, 1296 (2005).

${ }^{4}$ M. Kaniber, A. Kress, A. Laucht, M. Bichler, R. Meyer, M. Amann, and J. J. Finley, Appl. Phys. Lett. 91, 061106 (2007).

${ }^{5}$ I. S. Nikolaev, P. Lodahl, and W. L. Vos, Phys. Rev. A 71, 053813 (2005).

${ }^{6}$ K. J. Vahala, Nature (London) 424, 839 (2003).

${ }^{7}$ C. Simon, Y.-M. Niquet, X. Caillet, J. Eymery, J.-P. Poizat, and J.-M. Gérard, Phys. Rev. B 75, 081302(R) (2007).

${ }^{8}$ R. Sprik, B. A. van Tiggelen, and A. Lagendijk, Europhys. Lett. 35, 265 (1996).

${ }^{9}$ A. F. Koenderink, M. Kafesaki, C. M. Soukoulis, and V. Sandoghdar, J. Opt. Soc. Am. B 23, 1196 (2006).

${ }^{10}$ I. S. Nikolaev, P. Lodahl, A. F. van Driel, A. F. Koenderink, and W. L. Vos, Phys. Rev. B 75, 115302 (2007).

${ }^{11}$ J. R. Lakowicz, Principles of Fluorescence Spectroscopy (Springer, New York, 2006).

${ }^{12}$ J. Johansen, S. Stobbe, I. S. Nikolaev, T. Lund-Hansen, P. T. Kristensen, J. M. Hvam, W. L. Vos, and P. Lodahl, Phys. Rev. B 77, 073303 (2008).

${ }^{13}$ O. Labeau, P. Tamarat, and B. Lounis, Phys. Rev. Lett. 90, 257404 (2003).

${ }^{14}$ J. M. Smith, P. A. Dalgarno, R. J. Warburton, A. O. Govorov, K. Karrai, B. D. Gerardot, and P. M. Petroff, Phys. Rev. Lett. 94, 197402 (2005).

${ }^{15}$ L. C. Andreani and M. Agio, IEEE J. Quantum Electron. 38, 891 (2002). 